\title{
A Particle at Rest in a Static Gravitational Field
}

\author{
Nathan Rosen \\ University of North Carolina, Chapel Hill, North Carolina
}

\begin{abstract}
It is shown that, for a particle to be at rest in a static, axially symmetric gravitational field, the force on the particle must vanish. This result is then generalized to the case of an arbitrary static gravitational field.
\end{abstract}

\section{STATIC, AXIALLY SYMMETRIC FIELD}

$T^{\mathrm{H}}$ $\mathrm{HE}$ solutions of the Einstein gravitational field equations can be obtained for the case of a static, axially symmetric field by the method of Weyl ${ }^{1}$ and Levi-Civita. ${ }^{2}$ Using cylindrical polar coordinates $\rho, \varphi$, $z$ and time $t$, one takes for the line element

$$
d s^{2}=e^{2 \psi} d t^{2}-\rho^{2} e^{-2 \psi} d \varphi^{2}-e^{2 \gamma-2 \psi}\left(d \rho^{2}+d z^{2}\right),
$$

where $\psi$ and $\gamma$ are functions of $\rho$ and $z$. The gravitational field equations are satisfied if one chooses $\psi$ to satisfy the equation

$$
\left.\Delta \psi \equiv \underset{\rho}{-} \stackrel{1}{\rho}(\rho \psi, \rho)_{, \rho}+(\rho \psi, z), z\right]=0,
$$

while $\gamma$ is taken to satisfy the relations

$$
\gamma_{, \rho}=\rho\left[(\psi, \rho)^{2}-(\psi, z)^{2}\right], \quad \psi_{, z}=2 \rho \psi, \rho \psi_{, z} .
$$

A subscript preceded by a comma denotes a derivative with respect to the corresponding variable.

To get the Schwarzschild solution for the field of a single particle of mass $m$, one takes $\psi$ in the form of the Newtonian potential of a rod lying on the $Z$-axis, ${ }^{3}$ i.e. :

$$
\psi_{0}=\frac{1}{2} \ln \frac{r_{1}+r_{2}-2 m}{r_{1}+r_{2}+2 m}
$$

where $2 m$ is the length of the rod and $r_{1}$ and $r_{2}$ are the distances from the ends of the rod to the point in the field. Integration of Eqs. (3) gives for $\gamma$

$$
\gamma_{0}=\frac{1}{2} \ln \frac{\left(r_{1}+r_{2}\right)^{2}-4 m^{2}}{4 r_{1} r_{2}}
$$

However, in order to obtain the Schwarzschild solution in its usual, spherically symmetric form, it is necessary to carry out a transformation of the meridian plane. Taking, in place of $\rho$ and $z$, variables $r$ and $\theta$ according to the relations

$$
r=m+\frac{1}{2}\left(r_{1}+r_{2}\right), \quad \cos \theta=\left(r_{1}-r_{2}\right) / 2 m,
$$

one gets the Schwarzschild solution with spherical polar

1 H. Weyl, Ann. d. Physik 54, 117 (1918); 59, 185 (1919).

2 T. Levi-Civita, Rend. Acc. dei Lincei, 1919.

${ }^{3}$ R. Bach and H. Weyl, Math. Zeits. 13, 134 (1922).

$$
\text { coordinates }(r, \theta, \phi) \text { : }
$$

$$
\begin{aligned}
d s^{2}=-\left(1-\frac{2 m}{r}\right)^{-1} d r^{2}-r^{2}\left(d \theta^{2}+\sin ^{2} \theta d \phi^{2}\right) & \\
& +\left(1-\frac{2 m}{r}\right) d t^{2}
\end{aligned}
$$

The particle, previously represented by a rod, is now represented by a sphere $(r=2 m)$ on which the metric has a singularity.

Let us go back to the original coordinate system. To get a solution for a particle at rest in an external field having axial symmetry, one lets

$$
\psi=\psi_{0}+\chi,
$$

where $\chi$ also satisfies Eq. (2). One can consider $\chi$ as describing the external field. If one writes

$$
\gamma=\gamma_{0}+\delta
$$

then from Eqs. (3) one obtains

$$
\begin{aligned}
& \delta_{, \rho}=\rho\left[2 \psi_{0, \rho} \chi_{, \rho}-2 \psi_{0, z} \chi_{, z}+\left(\chi_{, \rho}\right)^{2}-\left(\chi_{, z}\right)^{2}\right], \\
& \delta_{, z}=2 \rho\left[\psi_{0, \rho} \chi_{, z}+\psi_{0, z} \chi_{, \rho}+\chi_{, \rho} \chi, z\right] .
\end{aligned}
$$

Let $\chi$ be well-behaved in the finite part of space, so that

$$
[\chi, \rho]_{\rho=0}=0 .
$$

From Eq. (10b) it follows that on the $Z$-axis $(\rho=0)$, if we measure $z$ from the midpoint of the rod, then

$$
\begin{array}{ll}
\delta_{, z}=0, & |z|>m, \\
\delta_{, z}=2 \chi_{,}, & |z|<m .
\end{array}
$$

It will be assumed that outside of the particle the field is free from singularities. Where there is no singularity, the space in each infinitesimal neighborhood must be Euclidean, so that for an infinitesimal circle, the ratio of circumference to diameter must be equal to $\pi$. For this to hold in the case of an infinitesimal circle with center on the $Z$-axis and lying in a plane perpendicular to the latter, one must have $\gamma=0$ on the $Z$-axis. This means that for $|z|>m, \rho=0$, one must have $\delta=0$. This can only be the case if, for $\rho=0$,

$$
\int_{-m}^{m} \delta, z d z=2\left(\chi_{z=m}-\chi_{z=-m}\right)=0 .
$$


If one interprets $\chi$ as the potential of the external gravitational field, this condition obviously means that the gravitational force acting on the particle vanishes. If one carries out the transformation (6), this condition continues to hold. We have then the result that, for a particle to remain at rest in an external gravitational field, the force on the particle must vanish. ${ }^{4}$

This result might have been expected on the basis of the fact that it has been shown that the equations of motion of a particle can be derived from the field equations. ${ }^{5}$ However, these proofs make use of expansions which require the assumption that the external field is weak, so that at a large distance from the particle the components of the metric tensor differ but slightly from their Galilean values. The field considered above was not subject to this restriction. To be sure, there were other restrictions, however: the field was static and axially symmetric.

\section{GENERAL CASE OF STATIC FIELD}

This raises the question as to the possibility of generalizing the result obtained in the previous section to the case of a static field that does not have axial symmetry. The difficulty with this more general case lies in the fact that no method has been found thus far for reducing the field equations to a linear form, corresponding to Eq. (2) above. Hence one cannot describe the fields by functions that combine by superposition. One therefore does not have any simple procedure for getting the solution of the field equations for a particle in an external field which would separate unambiguously the field of the particle from the external field.

By a simple transformation ${ }^{6}$ the expression for the line element near an isolated particle, given by Eq. (7), can be put into the isotropic form

$$
\begin{aligned}
d s^{2}=-\left(1+\frac{m}{2 r}\right)^{4}\left(d x^{2}+d y^{2}+\right. & \left.d z^{2}\right) \\
& +\left(\frac{1-(m / 2 r)}{1+(m / 2 r)}\right)^{2} d t^{2},
\end{aligned}
$$

with

$$
r^{2}=x^{2}+y^{2}+z^{2} .
$$

It is seen that the particle is now represented by the singularity on the sphere $r=m / 2$. The singularity consists in the vanishing of $g_{44}$, and hence of the determinant $g$, on the sphere.

Suppose now that this particle is at rest in a static external field. One would expect that, at sufficiently small distances from the particle, the field of the latter would become more important than the external field.

${ }^{4}$ The above discussion is a generalization of that in the paper by A. Einstein and N. Rosen, Phys. Rev. 49, 404 (1936). Also, see P. G. Bergmann, Introduction to the Theory of Relativity (Prentice-Hall, Inc., New York, 1942), p. 206.

5 A. Einstein, L. Infeld, and B. Hoffman, Ann. Math. 39, 65 (1938); A. Einstein and L. Infeld, Ann. Math. 41, 455 (1940).

${ }^{6}$ A. S. Eddington, The Mathematical Theory of Relativity (Cambridge University Press, London, 1924), p. 93.
Hence one can assume that, in a suitable coordinate system, the line element very close to the particle, i.e., for $r$ nearly equal to $m / 2$, is given by Eq. (13). For larger values of $r$, the line element will differ from this expression because of the external field. The procedure to be followed consists in expanding the field in the neighborhood of the particle in powers of $(r-m / 2)$ and investigating its behavior for small values of $(r-m / 2)$.

Accordingly, as a generalization of (13), we take for the components of the metric tensor the following expansions (Latin indices denoting 1, 2, 3):

$$
\left.\begin{array}{l}
g_{i k}=-\left(1+\frac{m}{2 r}\right)^{4}\left[\delta_{i k}-\left(r-\frac{m}{2}\right) a_{i k}-\cdots\right], \\
g_{4 k}=\left(1+\frac{m}{2 r}\right)\left(1-\frac{m}{2 r}\right)\left[0+\left(r-\frac{m}{2}\right) a_{4 k}+\cdots\right], \\
g_{44}=\left(1+\frac{m}{2 r}\right)^{-2}\left(1-\frac{m}{2 r}\right)^{2}\left[1+\left(r-\frac{m}{2}\right) a_{44}+\cdots\right],
\end{array}\right\}
$$

where the coefficients $a_{\mu \nu}$ are functions of $\left(\xi_{1}, \xi_{2}, \xi_{3}\right)$ with

$$
\xi_{k}=x_{k} / r,
$$

and where the form for $g_{4 k}$ has been chosen to give the same behavior for $g$ near $r=m / 2$ as in the case of (13).

Setting

$$
\lambda=r-(m / 2),
$$

and expanding all the functions of Eq. (14) in powers of $\lambda$, one gets

$$
\left.\begin{array}{l}
g_{i k}=16\left[-\delta_{i k}+\lambda\left(a_{i k}+\frac{4}{m} \delta_{i k}\right)+\cdots\right], \\
g_{4 k}=\frac{4 \lambda}{n}\left[0+\lambda a_{4 k}+\cdots\right], \\
g_{44}=\frac{\lambda^{2}}{m^{2}}\left[1+\lambda\left(a_{44}-\frac{2}{m}\right)+\cdots\right] .
\end{array}\right\}
$$

From these expressions, one gets for the components of the contracted Riemann-Christoffel tensor

$$
\left.\begin{array}{l}
R_{i k}=1 / 2 \lambda\left(-a_{i k}+\xi_{i} a_{k p} \xi_{p}+\xi_{k} a_{i p} \xi_{p}+2 \xi_{i} \xi_{k} a_{44}\right)+\cdots \\
R_{4 k}=0+\cdots, \\
R_{44}=\left(\lambda / 32 m^{2}\right)\left(a_{p p}-2 a_{p q} \xi_{p} \xi_{q}-2 a_{44}\right)+\cdots
\end{array}\right\}
$$

so that, in this approximation,

$$
R_{44}+\left(\lambda^{2} / 16 m^{2}\right) R_{p p} \equiv 0 .
$$

If one now takes as the field equations

$$
R_{\mu \nu}=0
$$

one finds for the solution

$$
a_{i k}=\xi_{i} A_{k}+\xi_{k} A_{i}-\xi_{i} \xi_{k} A, \quad a_{4 k}=B_{k}, \quad a_{44}=-\frac{1}{2} A,
$$

where $B_{k}$ and

$$
A_{k}=a_{k p} \xi_{p}
$$


are functions of $\left(\xi_{1}, \xi_{2}, \xi_{3}\right)$ and

$$
A=A_{q} \xi_{q}=a_{p q} \xi_{p} \xi_{q} .
$$

To get the force acting on the particle, one can surround the particle by a sphere of radius $r$ and center at the origin, and calculate the stress on the sphere as $r \rightarrow m / 2$. Using the gravitational stress-energy pseudotensor ${ }^{7} t_{\mu}{ }^{\nu}$, one finds that the stress acting across the surface of the sphere has components

$$
\mathfrak{t}_{k}{ }^{p} \xi_{p}=-\left(1 / 4 \pi m^{2}\right) \xi_{k}\left[8+m\left(a_{p p}-a_{p q} \xi_{p} \xi_{q}\right)\right]
$$

for $r=m / 2$. Using (20), one gets

$$
\mathfrak{t}_{k}^{p} \xi_{p}=-\left(2 / \pi m^{2}\right) \xi_{k} \text {. }
$$

Since the integral of (22a) over the sphere vanishes, it follows that the force on the particle vanishes.

This result represents the desired generalization. However, one can try to express it in terms of the external field in which the particle is located. This can be done provided, in Eq. (14), the series in the brackets are convergent for values of $r$ large compared to $m$. In that case, for such values of $r$, these equations become

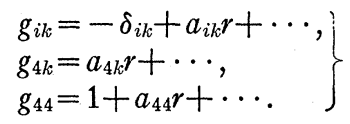

${ }^{7}$ See, for example, reference 6, p. 134.
Now, for $r$ large compared to $m$, one can expect the contribution of the particle to the field to be unimportant, so that Eq. (23) can be considered to describe the external field. If this external field were free from singularities in the absence of the particle, it would be expressed in the form

$$
g_{\mu \nu}=\left(g_{\mu \nu}\right)_{0}+\left(g_{\mu \nu, p}\right)_{0} \xi_{p} r+\cdots,
$$

where the subscript 0 denotes the value at the origin. Comparing with (23) we see that under these conditions it would be necessary to have

$$
a_{\mu \nu}=a_{\mu \nu p} \xi_{p},
$$

where the $a_{\mu \nu p}$ are constants. Going back to (20), we find that with this condition

$$
a_{i k}=a_{44}=0 .
$$

It follows that, for the metric associated with the external field, given by Eq. (23),

$$
\left\{\begin{array}{c}
\lambda \\
44
\end{array}\right\}_{0}=0
$$

which agrees with the equation of the geodesic for a particle at rest.

\title{
Remarks on Intra- and Inter-Molecular Migration of Excitation Energy
}

\author{
JAMES Franck \\ Department of Chemistry, University of Chicago, Chicago, Illinois \\ AND ROBERT LIVINGSTON \\ Department of Chemistry, University of Minnesota, Minneapolis, Minnesota
}

$\mathbf{M}$ IGRATION of excitation energy between molecules or inside a molecule from one of its groups to another is observed under many different conditions. The transferred excitation energy may be used just as the directly absorbed light energy for the promotion of chemical processes, or it may be dissipated into heat. The most remarkable facts are that the excitation energy under suitable conditions can be transferred with a high yield over distances of $\sim 50-100 \mathrm{~A}$ and that molecules or molecular groups which do not possess the quality to fluoresce still are able to transfer their excitation energy to energy acceptors which may not be in contact with them. There is no doubt that every example of transfer of electronic excitation energy observed so far can be understood either by an electron migration, or by exciton migration, or by the process of sensitized fluorescence, but in many cases uncertainty exists as to whether one of these three processes is solely responsible for a particular phenomenon and, if so, which one it is. In other cases experiences with migration of excitation energy are indiscriminately used as a proof for migration of energy in general. Especially, interpretations of energy transfer by and in substances of biological importance are often based on doubtful hypotheses. The present paper is a brief survey of the principles of the three processes by which migration of excitation energy can occur and gives examples of how a distinction between them can be reached.

Excitation energy in insulators may be transferred by electrons which by an act of light absorption are lifted 